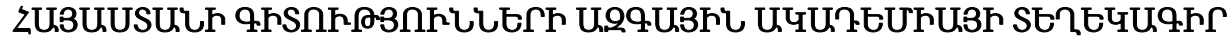 ИЗВЕСТИЯ НАЦИОНАЛЬНОЙ АКАДЕМИИ НАУК АРМЕНИИ
}

Uthaukipluw

68, №1, 2015

Механика

УДК 539.3

\section{SHEAR FLOQUET WAVES IN MAGNETO-ELECTRO-ELASTIC SOLID WITH PERIODIC INTERFACES OF IMPERFECT CONTACTS}

\section{Gasparyan D.K., Ghazaryan K.B.}

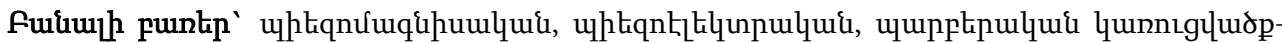
utin, uuhph unhpitip:

Ключевые слова: пьезомагнетик, пьезоэлектрик, периодическая структура, сдвиговые волны. Key words: piezoelastic, piezomagnetic, periodic structure, shear waves.

Гаспарян Д.К., Казарян К.Б.

Сдвиговые волны Флоке в магнитоэлектроупругих средах с периодическими поверхностями неполного контакта

В работе исследуется распространение сдвиговых волн в магнито-электро-упругих средах с одномерной периодической структурой поверхностей неполного контакта. В рамках теории Флоке получены дисперсионные уравнения, определяющие частотные зоны пропускания и задержки сдвиговых волн. Для трёх различных условий неполного контакта проведён анализ дисперсионных соотношений.

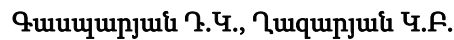

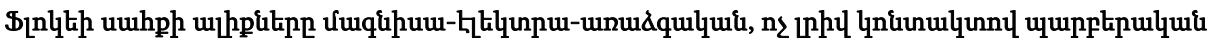

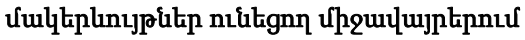

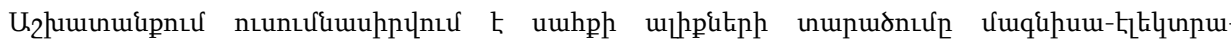

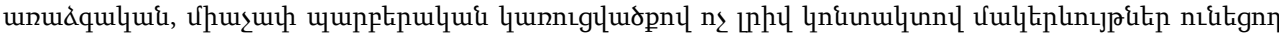

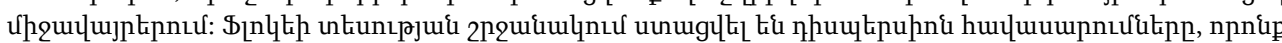

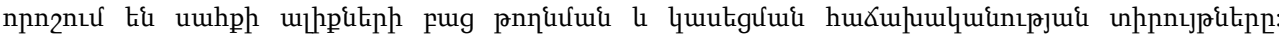

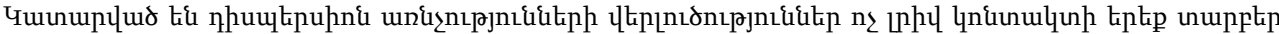

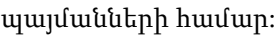

This paper aims at investigating the shear waves propagation in magneto-electro-elastic piezo active homogeneous solid of the one-dimensional periodic structure of imperfect contact interfaces. In the framework of the Floquet theory the dispersion equations are obtained defining shear wave frequency pass and gap band structure. For three kinds of imperfect contact conditions the analysis of dispersion relations is presented.

\section{Introduction}

The advent of new magneto-electro-elastic crystals (MEE) has enlarged the application fields of wave propagation in periodic media. Magneto-electro-elastic MEE crystals are one class of new composites that consist of piezoelectric and piezomagnetic phases. The magnetoelectric effect of piezoelectric-piezomagnetic composites was first reported in [1]. In the MEE crystal magnetoelectric effect is a coupled two field effect, in which the application of either a magnetic field or an electrical field induces an electrical polarization as well as a magnetization [1-4]. Investigations related to surface and bulk wave propagation 
in homogeneous , , multilayered structures made of MEE materials are presented in [5-11], where the quasi-static approximation of Maxwell equations was used [12]. The dispersion relations of SH waves in a heteroestructure with magneto-electro-elastic properties of $6 \mathrm{~mm}$ symmetry is studied in [5 ]. The propagation of Bleustein-Gulyaev surface wave is studied in [6] for transversely isotropic functionally graded MEE half-space. An analytical approach was used to investigate Love wave propagation in a layered MEE structure [7], where a solution of dispersion relations was obtained for magnetoelectrically open and short boundary conditions. In [8] the Rayleigh waves are investigated in MEE half plane. In [9] it is shown that shear surface waves with twelve different velocities in cases of different magnetoelectrical boundary conditions can be guided by the interface of two identical MEE half-spaces. The existence of shear surface wave travelling along the interface of two half spaces of different MEE materials is studied in [10]. The localized shear wave propagation is studied in [11] for MEE layer with quadratic and inverse quadratic inhomogeneity profiles of material parameters varying continuously along the layer thickness direction. A review of the most widely-used methods and approaches defining the Floquet waves in periodic multilayered structures are given in [12-13]. Dispersive behavior and band structure of SH waves in piezoelectric- piezomagnetic periodically layered structure are investigated in [14] .Within the full system of Maxwell's equations the effects of three kinds of imperfect contact transmission conditions on Floquet wave band gap structure are discussed in [15 ] for piezoelectric periodic structure.

The main goal of this work is related to the study of the behavior of shear Floquet waves in a magneto-electro-elastic homogeneous solid with periodically arranged 1D structure of imperfect interfaces structure. The following kinds of contacts are considered: electrically shorted, electromagnetically closed, sliding mechanical (lubricated) contacts. For all three kinds of contacts dispersion relations are derived. Typical numerical analyses of dispersion equations are presented and discussed for $\mathrm{MEE}$ solid made from $\mathrm{BaTiO}_{3}-\mathrm{CoFe}_{2} \mathrm{O}_{4}$.

This paper is organized as follows: In Section 2 we present the basic equations and relations for MEE solid. In Section 3 we derive the dispersion relations. While numerical analysis and discussion are given in Section 4, conclusions are drawn in Section 5.

\section{Governing equations and constitutive relations of MEE solid}

For a transversely isotropic piezo active magneto-electro-elastic (MEE) solid, polarized along $X_{3}$ direction, the stiffness, piezo-electromagnetic, magnetic, dielectric properties and bulk density can be represented by 15 independent coefficients $\left(c_{11}, c_{12}, c_{13}, c_{44}, c_{66}=\left(c_{11}-c_{12}\right) / 2\right)$, $\left(\beta_{13}, \beta_{15}, e_{13}, e_{15}, \alpha_{1}, \alpha_{3}\right),\left(\mu_{11}, \mu_{33}\right),\left(\varepsilon_{11}, \varepsilon_{33}\right), \rho$ correspondingly.

Consequently, the constitutive relations can be written in terms of the following expanded matrix form [ 5 ]: 


$$
\left(\begin{array}{l}
\sigma_{11} \\
\sigma_{22} \\
\sigma_{33} \\
\sigma_{23} \\
\sigma_{13} \\
\sigma_{12} \\
D_{1} \\
D_{2} \\
D_{3} \\
B_{1} \\
B_{2} \\
B_{3}
\end{array}\right)=\left(\begin{array}{cccccccccccc}
c_{11} & c_{12} & c_{13} & 0 & 0 & 0 & 0 & 0 & -e_{13} & 0 & 0 & -\beta_{13} \\
c_{12} & c_{11} & c_{13} & 0 & 0 & 0 & 0 & 0 & -e_{13} & 0 & 0 & -\beta_{13} \\
c_{13} & c_{13} & c_{33} & 0 & 0 & 0 & 0 & 0 & -e_{13} & 0 & 0 & -\beta_{13} \\
0 & 0 & 0 & c_{44} & 0 & 0 & 0 & -e_{15} & 0 & 0 & -\beta_{15} & 0 \\
0 & 0 & 0 & 0 & c_{44} & 0 & -e_{15} & 0 & 0 & -\beta_{15} & 0 & 0 \\
0 & 0 & 0 & 0 & 0 & c_{66} & 0 & 0 & 0 & 0 & 0 & 0 \\
0 & 0 & 0 & 0 & 0 & 0 & \varepsilon_{11} & 0 & 0 & g_{1} & 0 & 0 \\
0 & 0 & 0 & 0 & 0 & 0 & 0 & \varepsilon_{11} & 0 & 0 & g_{1} & 0 \\
e_{13} & e_{13} & e_{13} & 0 & 0 & 0 & 0 & 0 & \varepsilon_{33} & 0 & 0 & g_{3} \\
0 & 0 & 0 & 0 & 0 & 0 & g_{1} & 0 & 0 & \mu_{11} & 0 & 0 \\
0 & 0 & 0 & 0 & 0 & 0 & 0 & g_{1} & 0 & 0 & \mu_{11} & 0 \\
\beta_{13} & \beta_{13} & \beta_{13} & 0 & 0 & 0 & 0 & 0 & g_{3} & 0 & 0 & \mu_{33}
\end{array}\right)\left(\begin{array}{c}
s_{11} \\
s_{22} \\
s_{33} \\
2 s_{32} \\
2 s_{31} \\
2 s_{12} \\
E_{1} \\
E_{2} \\
E_{3} \\
H_{1} \\
H_{2} \\
H_{4}
\end{array}\right)
$$

Here $\sigma_{i j}$ are components of the stress tensor, $s_{i j}=\left(\partial_{i} u_{j}+\partial_{j} u_{i}\right) / 2$ are components of the elastic strain tensor , $E_{i}$ are components of the electrical field vector $\mathbf{E}, H_{i}$ are components of the magnetic field vector $\mathbf{H}, D_{i}$ are components of the electrical displacement vector $\mathbf{D}, \mathbf{B}_{i}$ are components of the magnetic induction vector $(i, j=1,2,3), u_{i}$ are components of elastic displacement vector, the indices preceded by a comma denote space-coordinate differentiation.

The interconnected elastic and electro-magnetic excitations in a MEE solid will be considered on the base of quasi-static approximation of Maxwell electrodynamics equations and linear equations of motion [16 ]

$\vec{\nabla} \times \mathbf{E}=0, \quad \vec{\nabla} \times \mathbf{H}=0, \quad \vec{\nabla} \cdot \mathbf{D}=0, \quad \vec{\nabla} \cdot \mathbf{B}=0$

$\partial_{i} s_{i j}=\rho \partial_{t t} u_{i}$

here $\vec{\nabla}=\left(\partial x_{1}, \partial x_{2}, \partial x_{3}\right)$.

In the case of a two dimensional problem (when $\partial / \partial x_{3}=0$ ) equations and relations separate into plane and anti-plane problems, analogous to the case of pure piezoelectric $6 \mathrm{~mm}$ symmetry crystal discussed in[17].

The anti-plane problem is described by the following equations and relations $\left(x_{1}=x, x_{2}=y\right)$

$\sigma_{x z}=G \partial_{x} u-e E_{x}-\beta H_{x} ; \quad \sigma_{y z}=G \partial_{y} u-e E_{y}-\beta H_{y} ;$

$D_{x}=e \partial_{x} u+\varepsilon E_{x}+g H_{x} ; \quad D_{y}=e \partial_{x} u_{z}+\varepsilon E_{y}+g H_{y} ;$

$B_{x}=\beta \partial_{x} u+g E_{x}+\mu H_{x} ; \quad B_{y}=\beta \partial_{y} u_{z}+g E_{y}+\mu H_{y} ;$ 
Here $\beta_{15}=\beta, e_{15}=e, c_{44}=G, u_{z}=U, \varepsilon_{1}=\varepsilon, \mu_{1}=\mu$

From (1) follows that

$$
E_{x}=-\partial_{x} \varphi ; \quad \vec{B}=-\vec{\nabla} \phi
$$

where $\varphi(x, y), \phi(x, y)$ are potential functions.

Taking into account (4) we can write relations (1) in the form

$$
\begin{aligned}
& \boldsymbol{\sigma}=\vec{\nabla}_{0}(G U+e \varphi+\beta \phi), \\
& \mathbf{B}_{\mathbf{0}}=\vec{\nabla}_{0}(\beta U-\mu \phi-g \varphi), \\
& \mathbf{D}_{0}=\vec{\nabla}_{0}(e U-\varepsilon \varphi-g \phi) ;
\end{aligned}
$$

Here the following notations are used

$$
\boldsymbol{\sigma}=\left(\sigma_{z x}, \sigma_{z y}\right), \mathbf{B}_{\mathbf{0}}=\left(B_{x}, B_{y}\right) ; \mathbf{D}_{0}=\left(D_{x}, \mathrm{D}_{y}\right) ; \vec{\nabla}_{0}=\left(\partial_{x}, \partial_{y}\right)
$$

Defining the new auxiliary potentials $[5,15]$

$$
F=\beta U-\mu \phi-g \varphi ; \quad S=e U-\varepsilon \varphi-g \phi
$$

and expressing potentials $\varphi, \phi$ via new potentials $F, S$

$\phi=(-F \varepsilon+S g+U \theta) \gamma^{-1} ; \quad \varphi=(-S \mu+U \eta+F) \gamma^{-1}$

$\gamma=\varepsilon \mu-g, \quad \theta=\beta \varepsilon-e g, \quad \eta=e \mu-\beta g$

we come to separate equations with respect to functions $u, F, S$.

$$
a^{2} \Delta u-\partial_{t t}^{2} u=0 ; \quad \Delta S=0 ; \quad \Delta F=0
$$

where the dot denotes time differentiation,
$\Delta \equiv\left(\partial_{x}^{2}+\partial_{y}^{2}\right) ; a^{2}=G_{0} / \rho ; G_{0}=G+(e \eta+\beta \theta) \gamma^{-1}, a$ is the velocity of shear bulk magneto-electro-elastic wave in the MEE solid.

Let us note that for transversely isotropic MEE crystal $\gamma>0$ [2].

The other functions $\sigma_{x z}, \sigma_{y z}, B_{x}, B_{y}, D_{x}, D_{y}$ via sought functions $u, F, S$ can be expressed as

$$
\begin{aligned}
& \sigma_{x z}=G_{0} \partial_{x} u-\gamma^{-1} \partial_{x}(\eta F+\theta S), \quad \sigma_{y z}=G_{0} \partial_{y} u-\gamma^{-1} \partial_{y}(\eta F+\theta S) ; \\
& B_{x}=\partial_{x} F ; \quad B_{y}=\partial_{y} F ; \quad D_{x}=\partial_{x} S ; \quad D_{y}=\partial_{y} S
\end{aligned}
$$

\section{Bloch-Floquet quasi-periodicity conditions, dispersion equations}

We consider the plane Bloch -Floquet wave propagation along arbitrary direction in $(x, y)$ plane of infinite homogenous MEE structure consisting of imperfect contact interfaces periodically arranged at points $x=n d, n=0, \pm 1, \pm 2 \ldots$. along $X$ direction. The effect of the interface periodicity on the dynamic behavior will be investigated, considering only the elementary cell $x \in(0, \mathrm{~d})$ and applying the Floquet-Bloch quasi-periodicity conditions 
connecting the ends of the elementary cell at $x=0, x=d[12,13]$.Solutions of equations (10) in $x \in(0, d)$ in the form of plane waves can be written as

$$
\begin{aligned}
& U(x, y, t)=\left[A_{1} \exp (\text { iqx })+A_{2} \exp (-i q x)\right] \exp [i(p y-\omega t)] \\
& F(x, y, t)=\left[A_{4} \exp (p x)+A_{2} \exp (-p x)\right] \exp [i(p y-\omega t)] \\
& S(x, y, t)=\left[A_{5} \exp (p x)+A_{6} \exp (-p x)\right] \exp [i(p y-\omega t)]
\end{aligned}
$$

Here, where the coefficients $A_{j}$ are unknown amplitudes of the waves, $q=\sqrt{\omega^{2} / a^{2}-p^{2}}$, $\omega$ is the angular frequency, $p$ is the wave number in $y$ direction, $i$ is the imaginary unit. We consider several types of partial imperfect contact transmission conditions at the interfaces within the periodically repeated unit cell of $d$ width (period): electrically shorted, electromagnetically closed, sliding mechanical (lubricated) contacts.

Note that electrically shorted conditions can be realized using a perfectly conducting film of negligible thickness; electromagnetically closed conditions can be realized using a perfectly conducting film of negligible thickness at the interfaces [15]. Smooth mechanical contacts correspond to lubricated interface conditions. The smooth mechanical contacts conditions were used in $[15,18]$ for piezoelectric layered structures.

\subsection{Electrically shorted interfaces with continuous elastic displacements, tractions, magnetic potential and magnetic induction}

Suppose that at each point $x=n d, n=0, \pm 1, \pm 2 \ldots$. there are electrically shorted interfaces where the tangential component of electrical field vector have vanished and normal component of electric displacement undergoes a discontinuity. Consequently the boundary conditions can be written as

$$
\begin{array}{lr}
\varphi(0, y, t)=0 ; \quad \varphi(d, y, t)=0 & \\
\sigma_{x}(0, y, t)=\lambda \sigma_{x}(d, y, t), & u(0, y, t)=\lambda u(d, y, t) \\
\phi(0, y, t)=\lambda \phi(d, y, t), & B_{x}(0, y, t)=\lambda B_{x}(d, y, t)
\end{array}
$$

Here and hereafter $\lambda=\exp (i k d), \mathrm{k}$ is the Floquet wave number.

Substituting solutions (11) into the Bloch-Floquet quasi-periodicity conditions and imperfect interface contact conditions(12) we come to the homogenous set of six simultaneous equations with respect to the unknown amplitudes $A_{j}$. The dispersion equation can be obtained by equating the determinants of the simultaneous sets of equations to zero, yielding

$$
\begin{aligned}
& \cos (d k)=F_{1}(\omega, p) ; \\
& F_{1}(\omega, p)=\frac{\left(K-K_{\beta}\right) p \cosh (d p) \sin (d q)-(1+K) q \cos (d q) \sinh (d p)}{\left(K-K_{\beta}\right) p \sin (d q)-(1+K) q \sinh (d p)}
\end{aligned}
$$

Here and hereafter the following notations are valid

$$
K_{\beta}=\frac{\beta^{2}}{G \mu} ; K_{e}=\frac{e^{2}}{G \varepsilon} ; K=\frac{K_{e}+K_{\beta}-2 \gamma_{0} \sqrt{K_{e} K_{\beta}}}{1-\gamma_{0}^{2}} ; \gamma_{0}=\frac{g}{\sqrt{\varepsilon \mu}}<1
$$


where $K_{e}$ is the electro-mechanical coupling coefficient, $K_{\beta}$ is the magneto-mechanical coupling coefficient.

\subsection{Electromagnetically closed interfaces with continuous elastic displacements and tractions}

Let suppose now that at each point $x=n d, n=0, \pm 1, \pm 2 \ldots .$. there are electromagnetically shorted interfaces where the tangential component of electric field vector, tangent component of magnetic field vector have vanished, while normal components of electric displacement and magnetic induction vectors undergo a discontinuity.

In this case the boundary conditions can be written as

$$
\begin{array}{ll}
\varphi(0, y, t)=\lambda \varphi(d, y, t) ; \phi(0, y, t)=\lambda \phi(d, y, t) \\
D_{x}(0, y, t)=\lambda D_{x}(0, y, t) ; B_{x}(0, y, t)=\lambda B_{x}(0, y, t) \\
\sigma_{x}(0, y, t)=0, \quad \sigma_{x}(d, y, t)=0 \\
\phi(0, y, t)=0 \quad \phi(d, y, t)=0
\end{array}
$$

The following dispersion equation corresponds to these conditions:

$\cos (d k)=F_{2}(\omega, p)$;

$F_{2}(\omega, p)=\frac{K p \cosh (d p) \sin (d q)-(1+K) q \cos (d q) \sinh (d p)}{K p \sin (d q)-(1+K) q \sinh (d p)}$

2.3 Mechanically sliding interface where normal elastic displacement undergo a discontinuity.

Let us suppose now that at each point $x=n d, n=0, \pm 1, \pm 2 \ldots .$. periodically arranged mechanically sliding interfaces where the component of electric field vector, tangent component of magnetic field vector have vanished, while normal components of electric displacement and magnetic induction vectors undergo a discontinuity.

In this case the boundary conditions can be written as

$$
\begin{aligned}
& \varphi(0, y, t)-\lambda \varphi(d, y, t)=0 ; \quad \phi(0, y, t)-\lambda \phi(d, y, t)=0 ; \\
& D_{x}(0, y, t)-\lambda D_{x}(0, y, t)=0 ; \quad B_{x}(0, y, t)-\lambda B_{x}(0, y, t)=0 \\
& \sigma_{x}(0, y, t)=0 ; \quad \sigma_{x}(d, y, t)=0
\end{aligned}
$$

The dispersion equation corresponding to these boundary conditions is the following

$$
\begin{aligned}
& \cos (d k)=F_{3}(\omega, p) \\
& F_{3}(\omega, p)=\frac{(1+K) q \cosh (d p) \sin (d q)+K p \cos (d q) \sinh (d p)}{(1+K) q \sin (d q)+K p \sinh (d p)}
\end{aligned}
$$

\section{Discussions and numerical results}

The dispersion equations $(13,15,17)$ defining ranges of frequencies associated with waves that can propagate in MEE solid (pass bands), alternated with ranges of frequencies corresponding

to waves that cannot be transmitted (stop or band gaps). 
For one dimension wave travelling along periodicity direction $(p=0)$ or when there are no piezo effects $\left(K_{e}=0, K_{b}=0\right)$, under electrically shorted or electromagnetically closed imperfect contact conditions from dispersion equations $(13,15)$ follows the simple dispersion relation $\omega=a k$; Under mechanically sliding condition instead of dispersion equation (17) we have $\cos (d k)=\cosh (d p)$, which means that the periodic structure does not allow propagation of elastic wave as was expected.

Let us now examine the behavior of the functions $F_{1}(\omega), F_{2}(\omega), F_{3}(\omega)$ the right parts of equations $(13,15,17)$. For given values of oblique incidence wave number $\mathrm{p}$ and frequency $\omega$ the dispersion equations have not real solution for $k$, when the right part of dispersion equations $|F(\omega, p)|>1$. The range of $\omega$ in which there are no propagating solutions of dispersions equations corresponds to complete photonic frequency band gap. Frequency regions of $\omega$ outside of gaps correspond to frequency passes .

Numerical calculation will be carried out for MEE crystal $\mathrm{BaTiO}_{3}-\mathrm{CoFe}_{2} \mathrm{O}_{4}$ for which dimensionless coupling coefficients are $K_{e}=0.24, K_{\beta}=0.36, \gamma_{0}=0,09$ [2].

The Fig.1, Fig2 illustrate dependence of right parts of dispersion equations $(15,17)$ with respect to dimensionless phase speed $\xi=\omega(p a)^{-1}$, for sliding and shorted interfaces, correspondingly, when $p d=2$. Points of intersection of function $F(\omega, p)$ with straight lines \pm 1 determine the frequency band and pass ranges.

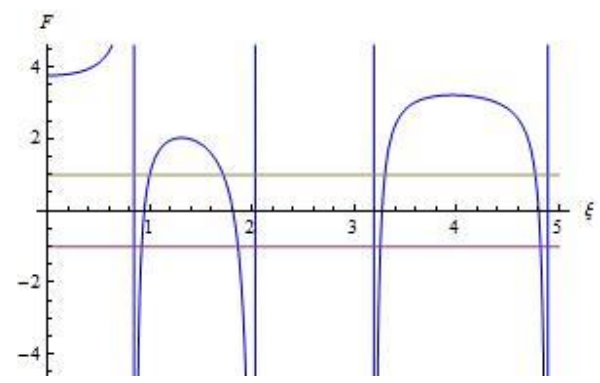

Fig1. The right part of Eq.(17) as function of dimensionless phase velocity $\xi$

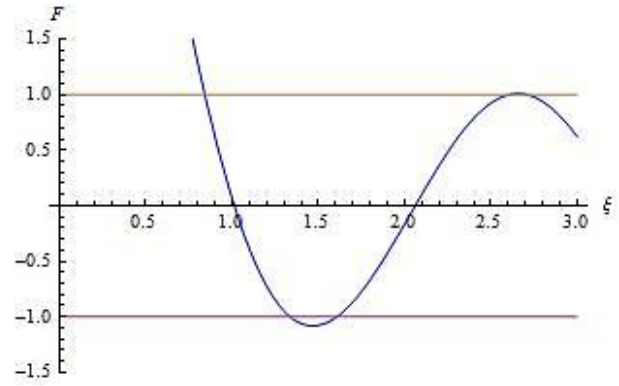

Fig 2. The right part of Eq.(13) as function of dimensionless phase velocity $\xi$

For sliding contact case from the Fig.1 follows that there are narrow ranges of frequency pass which means that periodically arranged interfaces of sliding contact may admit of shear wave transmission due to piezo effect. These passes are absent when the piezo effects are neglected. The layout of these pass ranges is $\xi \in(0 . \rightarrow 0.86), \xi \in(1.33 \rightarrow 1.64)$, $\xi \in(2.59 \rightarrow 2.76)$. The outside ranges correspond to the band gaps, the width of the band gaps significantly widens for higher frequencies.

Contrary to sliding contact case, for electromagnetically closed case from Fig.2 it follows that there are narrow ranges of gap zones caused by piezo effect. These gaps are absent when the piezo effects are neglected. The layout of these ranges are $\xi \in(0.90 \rightarrow 0.98), \xi \in(1.72 \rightarrow 1.86), \xi \in(3.22 \rightarrow 3.29), \xi \in(4.72 \rightarrow 4.79)$.

Outward of the gap ranges are the pass ranges, the width of which significantly widens for higher frequencies. 
For electrically shorted case Fig.3 shows the dispersions curves $k d=f\left(\omega d a^{-1}\right)$ and pass and stop bands structures in first Brillouin zone of dimensionless wave number $\mathrm{kd} \in(0, \pi)$, where $\omega d a^{-1}$ is the normalized dimensionless frequency. Dashed lines correspond to oblique wave number $p d=2$, solid lines to $p d=1$. The first and rest band gaps occur only at $\pi=\mathrm{kd}$. The width of the band gaps for MEE solids narrows for higher frequencies and gaps are absent when the piezo effects are neglected. The cut-off frequency are $0.89,1.86$ for $p d=1$, for $p d=2$, correspondingly.

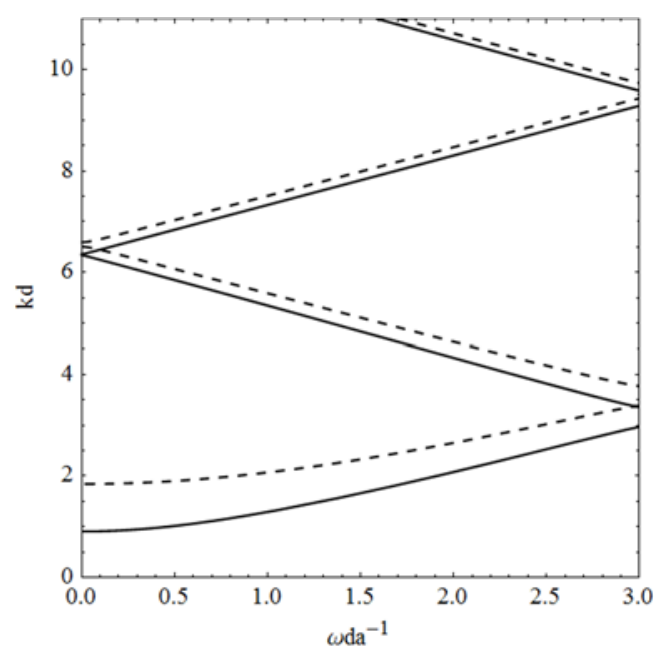

Fig 3. Dispersion curves for electrically shorted case

\section{Conclusions}

Based on quasi static approximation of Maxwell electrodynamics equations shear wave propagation in MEE homogeneous solid is studied. The well known Floquet quasiperiodicity boundary conditions are taken into consideration. Dispersion relations are derived for three kinds of imperfect contacts: electrically shorted, electromagnetically closed, sliding mechanical (lubricated) contacts. For MEE crystal $\mathrm{BaTiO}_{3}-\mathrm{CoFe}_{2} \mathrm{O}_{4}$ the numerical analysis of dispersion equations determining the Floquet waves is carried out. The Floquet wave frequency pass and gap band structures are studied in conformity with contact conditions. The numerical results estimating effects of transmission conditions and piezo effects are presented. The results show that for periodically arranged electrically shorted or electromagnetically closed interfaces the possibility of frequency band gap is conditioned by piezo effect. It is also shown that periodically arranged mechanically sliding interfaces may admit shear wave transmission/ pass, which simply do not exist without a piezo effect.

\section{References}

1. J.van Suchtelen, Product properties: a new application of composite materials. //Philips Res Rep, v.27, 1972, pp. 28-37.

2. C.W. Nan, Magnetoelectric effect in composites of piezoelectric and piezomagnetic phases. // Physical Review B, v.50, 1994, pp. 6082-6088.

3. Benveniste, Y.,. Magnetoelectric effect in fibrous composites with piezoelectric and piezomagnetic phases. //Physical Review B, v.51, 1995, pp.16424-16427. 
4. P. Kondaiah, K. Shankar, N. Ganesan, Pyroelectric and pyromagnetic effects on behavior of magneto-electro-elastic plate.// Coupled Systems Mechanics, v.2, №1, 2013, pp.1-22.

5. H. Calas, J.A. Otero, R. Rodrı'guez-Ramos, G. Monsivais, C. Stern, Dispersion relations for $\mathrm{SH}$ wave in magneto-electro-elastic heterostructures. // International Journal of Solids and Structures, v.45, 2008, pp. 5356-5367.

6. P. Li, F. Jin, Z.Qian, Propagation of the Bleustein-Gulyaev waves in a functionally graded transversely isotropic electro-magneto-elastic half-space. // European Journal of Mechanics A/Solids, 37, 2013, pp.17-23.

7. J.K. Du, X.Y. Jin, J. Wang, Love wave propagation in layered magnetoelectro-elastic structures. Science in China Series G: Physics, Mechanics \& Astronomy, v. 51,2008, pp. 617-631.

8. W.J. Feng, E. Pan, X. Wang, J. Jin, Rayleigh waves in magnetoelectro-elastic half planes. // Acta Mech., 2008, v.202, pp.127-134.

9. Melkumyan, Twelve shear surface waves guided by clamped/free boundaries in magneto-electro-elastic materials. // Journal of Solids and Structures, v.44, 2007, pp.3594-3599.

10. D. Piliposyan, Shear surface waves at the interface of two magnetoelectro-elastic media. //Multidiscipline Modelling in Materials and Structures, v.8, 2012, pp. 417-426.

11. D. Gasparyan., K. Ghazaryan, Shear Waves in Functionally Graded Electro-MagnetoElastic Media. // International Journal of Engineering Research \& Technology (IJERT), 3,10, 2014, pp.769-776.

12. J. Gazalet, S. Dupont, J.C. Kastelik, Q. Rolland, B. Djafari-Rouhani. A tutorial survey on waves propagating in periodic media: Electronic, photonic and phononic crystals. Perception of the Bloch theorem in both real and Fourier domains, Wave Motion, 50, 2013, pp. 619-654

13. A.Maurel, P.A.Martin, V.Pagneux, Effective propagation in a one-dimensional perturbed periodic structure:comparison of several approaches, Waves in Random and Complex Media, v. 20, 4, 2010, pp.634-6557

14. Yu Pang, Jin-Shan Gao, Jin-Xi Liu, SH wave propagation in magnetic-electric periodically layered plates, Ultrasonics, 54, (2014) pp.1341-1349

15. K.B Ghazaryan, D.G Piliposyan, Interfacial effects for shear waves in one dimensional periodic piezoelectric structure. // Journal of Sound and Vibration, 330 (26), 2011, pp. 6456-6466.

16. С.А.Амбарцумян, М.В.Белубекян, Некоторые задачи электромагнитоупругости пластин Ереван: Изд. Ереванского университета, 1991.143 с.

17. А.Аветисян, К задаче распространения сдвиговых волн в пьезоэлектрической среде. // Изв.НАН Армении. Механика. 1985. Т.38. №1. С.12-19.

18. M.V. Belubekyan, V.M. Belubekyan, Surface waves in piezoactive elastic system of a layer on a semi-space. Proc. of the Yerevan State University., Phys.\& Math. Sci., 2013, 3, pp.45-48.

19. Mei-Feng Liu, An exact deformation analysis for the magneto-electro-elastic fiberreinforced thin plate. Applied Mathematical Modelling, 35, 2011, pp.2443-2461.

\section{Сведения об авторах:}

Казарян Карен Багратович - доктор физ.-мат.наук, профессор, Главный научный сотрудник Института механики НАН Армении E-mail: ghkaren@gmail.com

Гаспарян Давид Каренович - аспирант

E-mail: david.gasparyan@yahoo.com

Поступила в редакцию 09.02.2015 